\title{
V 1500 Cyg: SLOW VARIABILITY IN POST-NOVA STAGE
}

\author{
B. Pavlenko \\ Crimean Astrophysical Observatory \\ 334413 p/o Nauchny, Crimea, USSR
}

Regular photometric observations of V1500 Gyg (Nova Cygni 1975) have been carried out at the Crimean Astrophysical Observatory of the USSR Academy of Sciences from 1975 till now. The $0.5 \mathrm{~m}$ telescope equipped with a TV system is used to estimate the star brightness in v.

The decline of the mean brightness is presented in Figure 1 . The solid lines (1) and (2) show the changes of the $V$ brightness according to Patterson (1979) and Arkhipova and Zaitseva (1978), respectively. The dotted line is drawn according to our data (Pavlenko 1988). The arrows indicate the amplitudes of the light and colour variations.

In addition to a well known main period $P_{0}=0.41396131$, a slow variability of V1500 Cyg was noticed even in 1978 (Abramenko and Pavlenko 1983). The existence of this variability was confirmed in 1978-1987 (Pavlenko and Pelt 1988). In Figure 2 a low resolution power spectrum (the fading of the nova and the harmonic components of the main period were removed) is plotted against frequency. The most powerful minimum in the region $5-20$ days corresponds to $P_{1}=7.070$. The refined spectrum for the region around $P_{1}$ shows several peaks $P_{1}=7.07019, P_{2}=7 . d 7352, P_{3}=7 . d 7731$, etc. The value of $P_{3}$ is closer to the "beat" period between the main photometrical (orbital) $P_{0}$ and polarimetrical $P_{p_{01}}=0 .{ }^{a} 137154$ (Stockman et al 1988).

In Figure 3 the orbital light curves 1980-1985 are sampled with the phase $\psi$ of the period $P_{3}=7.48$. One can see that the shape of the light curves changes with this period: a) in "high" stages of the nova brightness $(\psi=0.4$ to 0.6$)$ the curves show a broad maximum; b) in the intermediate stage $(\psi=0.7$ to 0.9$)$ vice versa, there is a broad quasi-flat minimum. The curves " $c "(\psi=0.9$ to 0.1$)$ and " $d "$ $(\psi=0.2$ to 0.4$)$ are characterized by a rapid increase of brightness, rather than by a decrease.

The change of the shape of the light curves may be caused by different effects connected with variable orientation of the magnetic axis of the white dwarf with respect to the red one. 


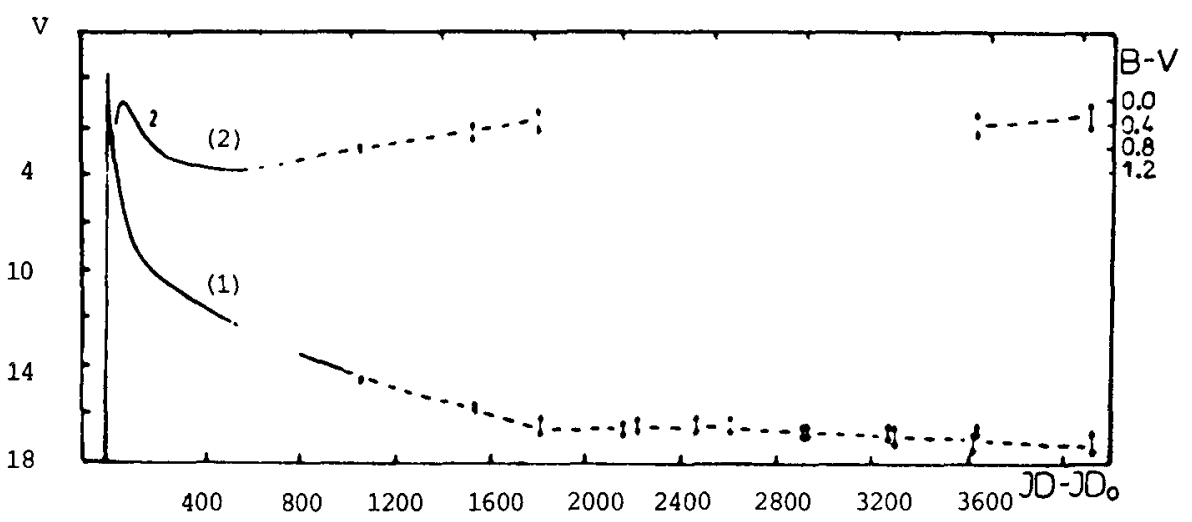

Figure 1: Variations on the mean brightness and colour index B-V of V1500 Cyg from 1975 to 1986. Abscissae are in days after maximum light.

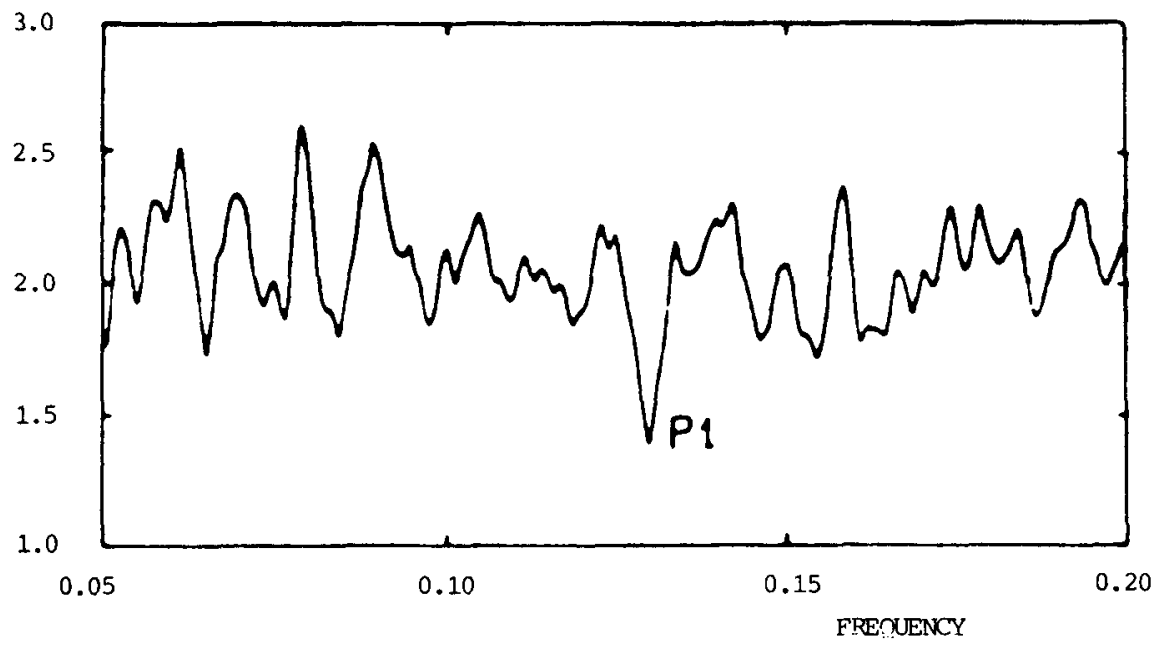

Figure 2: Power spectrum of VI500 Cyg in the interval 5 - 20 days. 


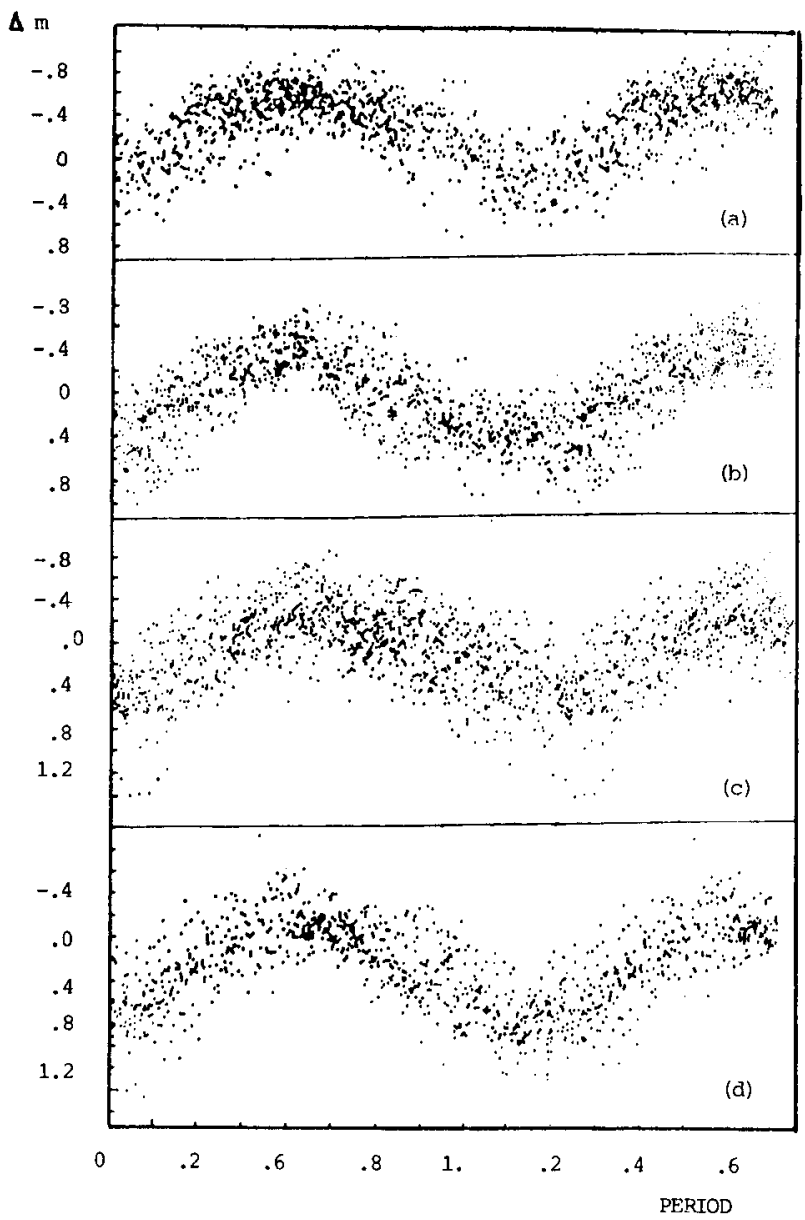

Figure 3: Variations of orbital light curves in different phases of the $7 . d 8$ period: a) $\psi=0.4$ to $0.6, \quad$ b) $\psi=0.7$ to 0.9 , c) $\psi=0.9$ to 0.1 , d) $\psi=0.2$ to 0.4 . The brightness of the nova is reduced to the mean value.

\section{REFERENCES}

Patterson, J.:1979, Astrophys. J., 131, 789 .

Arkhipova, V.P. and Zaitseva, G.V.:1978, Astron. Tsirk., 1005, 4

Pavlenko, E.P.:1988, Bull. Crimean Astrophys. Obs., 79, 103 .

Abramenko, A.N. and Pavlenko, E.P.:1983, Bull. Crimean Astrophys. Obs. 66,183 .

Pavlenko, E.P., Pelt, J.:1988, JBVS, No. 3252.

Stockman, H.S., Schmidt, G.D., Lamb, D.Q.:1988, Astrophys. J., 1 